\section{Hoher Nutzen in Bezug auf die Lebensqualität}

Ziel der vorliegenden systematischen Literaturübersicht und Metaanalyse war es, den Einfluss der Implantation einer Knietotalendoprothese auf die mittel- und langfristige postoperative gesundheitsbezogene Lebensqualität (postoperative health-related quality of life - HRQOL) zu untersuchen.

Shan L, Shan B, Suzuki et al. Intermediate and long-term quality of life after total knee replacement: a systematic review and meta-analysis. J Bone Joint Surg Am 2015; 97: $156-168$

\section{Einleitung \\ $\nabla$}

Die Endoprothetik wurde in den letzten Jahren, bei deutlich steigenden Implantationszahlen, vermehrt in den Fokus der öffentlichen Diskussion gebracht. Berichte über viele unzufriedene Patienten bei der Knieendoprothetik haben die allgemeine Diskussion über unnötige Operationen angefacht. In diesem Zusammenhang ist aufgrund der sich verändernden Erwartungen an das Ergebnis des Eingriffes und den Wunsch nach einem aktivem Lebensstil, die patientenbezogene Untersuchung der Lebensqualität im Rahmen der Erfassung der Ergebnisse immer wichtiger. Die HRQOL stellt dabei mittlerweile eines der Schlüsselziele zur Erfassung des postoperativen Ergebnisses dar.

\section{Methodik}

\section{$\nabla$}

Es wurden alle publizierte Studien seit Januar 2000 mit primärer Implantation ei-

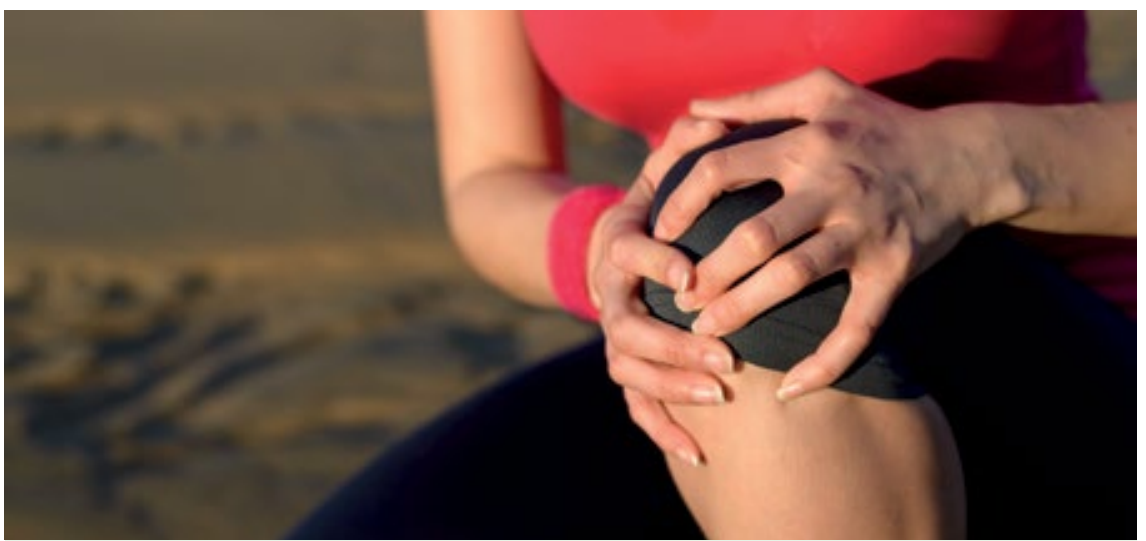

Shan et al. untersuchten die Patientenzufriedenheit nach Knietotalendoprothese (Bild: Dirima / Fotolia.com). ner Knietotalendoprothese bei Diagnose einer Gonarthrose und einem Mindestnachuntersuchungszeitraum von 3 Jahren betrachtet. Die Schlüsselzielkriterien waren die postoperative Lebensqualität in Bezug auf Schmerz, Funktion und Zufriedenheit im Vergleich zum präoperativen Zustand. Bei strikten Ein- und Ausschlusskriterien wurden nur Studien, welche krankheitsspezifische und/oder allgemeine HRQOL-Daten berichteten, eingeschlossen.

\section{Ergebnisse}

19 Studien mit Erfassung von 5110 Patienten wurden in die Auswertung einbezogen. Das typische Patientenalter (mean oder median) zum Zeitpunkt der Operation lag bei 63,8-92 Jahren. Der Nachuntersuchungszeitraum (mean oder median) lag bei 3-21 Jahren. Die meisten Studien $(\mathrm{n}=16)$ waren prospektiv, $\mathrm{n}=13$ Studien hatten den Evidenz-Level 1 oder 2.
In der Zusammenfassung der Bereiche „Schmerz“ und „Funktion“ des WOMAC (Western Ontario and McMaster Universities Osteoarthritis Index) und KSS (Knee Society Score), konnte ein ausgeprägter positiver Einfluss der Knieprothesenimplantation im Vergleich zum präoperativen Zustand festgestellt werden. Der Therapieeffekt beider Bereiche war $>1,0$ (In der Endoprothetik weist ein Effekt $>0,8$ auf eine sehr effektive Therapie hin).

Mehr als 75\% der Patienten waren zufrieden oder sehr zufrieden mit dem Operationsergebnis. 76\% konnten ohne Hilfsmittel laufen, ohne oder mit leichtem Hinken. Die Gehdistanz und die Fähigkeit Treppen zu steigen war deutlich verbessert. 79,5\% würden den Eingriff noch einmal durchführen lassen.

\section{Kommentar \\ $\nabla$}

Die vorliegende systematische Literaturübersicht und Metaanalyse liefert aufgrund der Fokussierung des Effektes der Knieprothesenimplantation auf die mittel- und langfristige HRQOL einen wichtigen Beitrag für die momentan geführten Diskussionen über die Wirksamkeit dieser operativen Therapie. Unstrittig stellt sich dabei der positive Effekt der postoperativen Verbesserung in Bezug auf die Bereiche des Schmerz und der Funktion dar. Der allgemeine Gesundheitszustand verbesserte sich allerdings nicht, was wahrscheinlich dem natürlichen Alterungsprozess geschuldet ist. Kritisch muss man sehen, dass „nur“ 3 von 4 Patienten zufrieden sind und dass „nur“ knapp 80\% der Patienten den Eingriff noch einmal durchführen lassen würden. Anspruch und Wirklichkeit scheinen letztendlich trotz der Verbesserung von Schmerz und Funktion noch zu häufig nicht im Einklang zu stehen. Eine kritische Indikationsstellung und Aufklärung der Patienten über das zu erwartende Ergebnis im jeweils individuellen Fall sollte die Zufriedenheitsrate erhöhen sowie die allgemeine Sichtweise auf die Knieendoprothetik verbessern können.

PD Dr. med. Christoph Becher Orthopädische Klinik der Medizinischen Hochschule Hannover christoph.becher@ddh-gruppe.de 